Surveys in Differential Geometry XIV

\title{
Harmonic mappings and moduli spaces of Riemann surfaces
}

\author{
Jürgen Jost and Shing-Tung Yau
}

\section{Introduction}

Harmonic maps constitute a powerful tool for the analysis of moduli and Teichmüller spaces of compact Riemann surfaces. These moduli and Teichmüller spaces parametrize the different conformal structures a given compact differentiable surface $F$ of some genus $p$ can carry, together with a topological marking in the Teichmüller case. That is, each point in the moduli space $M_{p}$ or the Teichmüller space $T_{p}$ corresponds to some (marked) Riemann surface of genus $p$, that is, to some conformal structure on $F$. $M_{p}$ then is a quotient of $T_{p}$ by the mapping class group $\Gamma_{p}$, the group of homotopy classes of oriented diffeomorphisms of $F$. Since some nontrivial elements of $\Gamma_{p}$ have fixed points, $M_{p}$ acquires some quotient singularities. However, a suitable finite covering of $M_{p}$ is free from singularities, that is, a manifold. Therefore, for many aspects, the singularities of $M_{p}$ can be ignored, and this will sometimes simplify our discussion. - In this review, we shall confine ourselves mostly to the case $p \geq 2$ which is the most difficult and most interesting case.

Harmonic maps come from Riemannian geometry. They are defined as maps $h: M \rightarrow N$ between Riemannian manifolds that minimize a certain variational integral, called the energy. Thus, they depend on the Riemannian metrics of $M$ and $N$. The theory works best when the metric of $N$ has nonpositive sectional curvature.

Harmonic maps can then be applied to moduli or Teichmüller spaces in two different ways. On one hand, one can look at harmonic maps to, from, or between Riemann surfaces and study how the harmonic maps or quantities associated to them, like their energy, depend on the underlying conformal structures. This is facilitated by the fact that the harmonic maps in question are unique in their homotopy classes, as we are looking at the case of genus $p \geq 2$ which implies that the Riemann surfaces can be equipped with a hyperbolic metric, that is, one with constant negative curvature. In that manner,

(C)2009 International Press 
we can then produce functions on $M_{p}$ or $T_{p}$, like the energy of the harmonic map as a function of the Riemann surface on which it is defined. We can then derive properties of these functions that lead to conclusions about the topology or geometry of $M_{p}$ or $T_{p}$. This scheme provides an alternative to schemes that depend on other types of mappings, like quasiconformal ones.

On the other hand, when we study families of Riemann surfaces, we obtain induced mappings into $M_{p}$ or $T_{p}$. These mappings then are naturally holomorphic, and thus in particular harmonic. So, one can apply general theorems about harmonic mappings to obtain restrictions on families of Riemann surfaces, like finiteness results. Conversely, when we have some mapping into $M_{p}$ or $T_{p}$, not necessarily holomorphic, we can appeal to general existence theorems about harmonic maps to deform it into a harmonic map. Here, again, we need to use some negative curvature. This time, it is provided by the Weil-Petersson on $T_{p}$ and on its quotients, which is a Kähler metric of negative sectional curvature. In fact, the curvature properties of the Weil-Petersson metric are such that general results apply to show that harmonic maps into $T_{p}$ or $M_{p}$ of sufficiently high rank have to be even holomorphic (or antiholomorphic, but that case is usually of less interest). That is, from topological conditions alone one can produce holomorphic maps into $M_{p}$. These holomorphic maps then correspond to families of Riemann surfaces.

Also, while a harmonic map to a Riemann surface in general is not holomorphic - which simply reflects the fact that we can deform the underlying conformal structure -, nevertheless, a harmonic map from a higher dimensional compact Kähler manifold $M$ to a hyperbolic Riemann surface $S$ induces some holomorphic foliation on $M$. This was our original discovery many years ago which was a starting point for our subsequent research and, in fact, for the entire field of non-abelian Hodge theory. In particular, one can take the quotient of $M$ by this holomorphic foliation. That quotient then also is a compact Riemann surface $S^{\prime}$ that is a covering of the original target $S$. Thus, while the original harmonic map to $S$ need not be holomorphic, he quotient map from $M$ to $S^{\prime}$ then is holomorphic by construction (the induced map from $S^{\prime}$ to $S$ is harmonic, but need not be holomorphic).

After summarizing background material about harmonic maps and moduli spaces of Riemann surfaces, this review will then explore these two principles, that is, harmonic maps of Riemann surfaces, and harmonic maps into moduli or Teichmüller spaces.

For a more extensive review with a somewhat different emphasis, we refer to $[\mathbf{1 3}]$. In particular, that review contains a more extended bibliography than ours. We also thank Michael Wolf and the referees for useful comments and references.

\section{Background}

\subsection{Harmonic maps.}

2.1.1. Equivariant harmonic maps. Since the theory of harmonic mappings is systematically presented in $[\mathbf{4 4 , 4 6}$, we can be brief here. Since we 
shall have to cope with spaces that possess certain singularities, we shall need existence results for harmonic mappings that are formulated on universal covering spaces in an equivariant manner. Therefore, we shall present here the existence theorem of Jost-Yau [36] and Labourie [49] for equivariant harmonic maps. The setting of this result is the following. Let $X, Y$ be simply connected Riemannian manifolds with isometry groups $I(X), I(Y)$. $Y$ will always be assumed to be complete. Let $\Gamma$ be a discrete subgroup of $I(X)$. The quotient $X / \Gamma$ then may have singularities, arising from fixed points of nontrivial elements of $\Gamma$. Nevertheless, it inherits a metric and a volume form $d \mu$ from $X$, and we shall identify it with a fundamental region for the action of $\Gamma$. When $\Gamma$ acts without fixed points and properly discontinuously, then the quotient $M:=X / \Gamma$ is a Riemannian manifold itself. We now assume that we have a homomorphism

$$
\rho: \Gamma \rightarrow I(Y)
$$

and we are interested in $\rho$-equivariant maps, that is, maps $f: X \rightarrow Y$ satisfying

$$
f(\gamma x)=\rho(\gamma) f(x) \text { for all } \gamma \in \Gamma \text { and } x \in X .
$$

In particular, when $\Gamma$ operates without fixed points and properly discontinuously, $\Gamma$ then can be identified with the fundamental group $\pi_{1}(M)$. In other words, we have constructed a setting where we can investigate representations of fundamental groups $\pi_{1}(M)$ in isometry groups $I(Y)$ of simply connected Riemannian manifolds. An important example is the case where $Y=G / K$ is a symmetric space of noncompact type, in which case $G$ is a linear algebraic group with maximal compact subgroup $K$. In this case, $G$ is the identity component of $I(Y)$, and so, we look at representations of $\Gamma$ in linear algebraic groups.

When $\rho(\Gamma)$ acts fixed point free and properly discontinuously, then $I(Y) /$ $\rho(\Gamma)$ is a Riemannian manifold. Thus, when both $\Gamma$ and $\rho(\Gamma)$ share this property, then the representation $\rho$ corresponds to a homotopy class of maps between the Riemannian manifolds $M:=X / \Gamma$ and $N:=Y / \rho(\Gamma)$. We can also look at subgroups $\Lambda \in I(Y)$ with $\rho(\Gamma) \in \Lambda$ and then look at maps from $M$ to $I(Y) / \Lambda$. In particular, in the above situation, we may consider quotients of $N$.

We now want to optimize the map, and it is at this point that we need the Riemannian structures of $X$ and $Y$. The distance function of $Y$ induced by the Riemannian metric will be written as $d(.,$.$) . We also recall some geo-$ metric notation (see $[\mathbf{4 6}]$ ). Let $m:=\operatorname{dim} X, n:=\operatorname{dim} Y$. In local coordinates, the metric tensor of $X$ is written as

$$
\left(\gamma_{\alpha \beta}\right)_{\alpha, \beta=1, \ldots, m}
$$

and the one of $Y$ as

$$
\left(g_{i j}\right)_{i, j=1, \ldots, n}
$$


Some further notation, with the standard summation convention:

$$
\begin{array}{ll}
\left(\gamma^{\alpha \beta}\right)_{\alpha, \beta=1, \ldots, m}=\left(\gamma_{\alpha \beta}\right)_{\alpha, \beta}^{-1} & \text { (inverse metric tensor) } \\
\gamma:=\operatorname{det}\left(\gamma_{\alpha \beta}\right) & \\
\Gamma_{j k}^{i}:=\frac{1}{2} g^{i \ell}\left(g_{j \ell, k}+g_{k \ell, j}-g_{j k, \ell}\right) & \text { (Christoffel symbols of } Y) .
\end{array}
$$

The volume form of $X, \sqrt{\gamma} d x^{1} \cdots d x^{m}$ in local coordinates, is abbreviated as $\operatorname{dvol}(x)$ or $d X$.

The Laplace-Beltrami operator of $X$ is

$$
\Delta_{X}=-\frac{1}{\sqrt{\gamma}} \frac{\partial}{\partial x^{j}}\left(\sqrt{\gamma} \gamma^{\alpha \beta} \frac{\partial}{\partial x^{i}}\right)
$$

Sometimes, it will be more convenient to use the abstract intrinsic formalism of Riemannian geometry. The metric of a vector bundle $E$ over $X$ will be denoted as $\langle\cdot, \cdot\rangle_{E}$. If $f: X \rightarrow Y$ is a $\rho$-equivariant map of class $C^{1}$, we define its energy density in local coordinates $\left(x^{1}, \ldots, x^{m}\right)$ on $X,\left(f^{1}, \ldots, f^{n}\right)$ on $Y$ as

$$
e(f)(x):=\frac{1}{2} \gamma^{\alpha \beta}(x) g_{i j}(f(x)) \frac{\partial f^{i}(x)}{\partial x^{\alpha}} \frac{\partial f^{j}(x)}{\partial x^{\beta}} .
$$

$e(f)(x)$ is an intrinsic quantity, independent of the choices of local coordinates. With $\frac{\partial f}{\partial x^{\alpha}}=\frac{\partial f^{i}}{\partial x^{\alpha}} \frac{\partial}{\partial f^{i}}$ and $d f=\frac{\partial f^{i}}{\partial x^{\alpha}} d x^{\alpha} \otimes \frac{\partial}{\partial f^{i}}$, considered as a section of the bundle $T^{*} X \otimes f^{-1} T Y$, we have

$$
\begin{aligned}
e(f) & =\frac{1}{2} \gamma^{\alpha \beta}\left\langle\frac{\partial f}{\partial x^{\alpha}}, \frac{\partial f}{\partial x^{\beta}}\right\rangle_{f^{-1} T Y} \\
& =\frac{1}{2}\langle d f, d f\rangle_{T^{*} X \otimes f^{-1} T Y} \\
& =\frac{1}{2}\|d f\|^{2},
\end{aligned}
$$

where the norm $\|\cdot\|$ involves the metrics on $T^{*} X$ and $f^{-1} T Y$.

Definition 1. The energy of a $\rho$-equivariant $C^{1}$-map $f: X \rightarrow Y$ is

$$
E(f):=\int_{X / \Gamma} e(f) d X
$$

where we integrate over a fundamental region for the action of $\Gamma$, as explained.

$A$ (smooth) map $h$ that minimizes the energy $E$ among all $\rho$-equivariant maps is called harmonic.

We remark here that in general all critical points of the energy functional, and not only the minimizers, are called harmonic. In the context of the present survey, however, only minimizing harmonic maps will occur. 
A harmonic map $h$ has to satisfy the nonlinear elliptic system

$$
\begin{aligned}
& \frac{1}{\sqrt{\gamma}} \frac{\partial}{\partial x^{\alpha}}\left(\sqrt{\gamma} \gamma^{\alpha \beta} \frac{\partial}{\partial x^{\beta}} h^{i}\right)+\gamma^{\alpha \beta}(x) \Gamma_{j k}^{i}(h(x)) \frac{\partial}{\partial x^{\alpha}} h^{j} \frac{\partial}{\partial x^{\beta}} h^{k}=0 \\
& \quad \text { for } i=1, \ldots, n,
\end{aligned}
$$

or in more condensed notation

$$
\Delta_{X} h-\Gamma(d h, d h)=0 .
$$

When the domain is one-dimensional, harmonic maps are nothing but geodesics in $Y$, or in the quotient case, closed geodesics considered as maps $S^{1} \rightarrow N$. Of course, it is well known that every homotopy class of maps from $S^{1}$ into a compact Riemannian manifold $N$ contains a closed geodesic.

It turns out that in higher dimensions, one needs to assume that $Y$ has nonpositive sectional curvature to get a good regularity theory that ensures the smoothness of weak solution of (2.8). For the existence, one then can try to minimize the energy among all $\rho$-equivariant maps. In order to obtain the convergence of a minimizing sequence, one needs in addition a topological condition that we are now going describe.

A representation $\rho: \Gamma \rightarrow I(Y)$ is called reductive if there exists a totally geodesic subspace $Y^{\prime}$ of $Y$ stabilized by $\rho(\Gamma)$ with the property that for every totally geodesic subspace $Y^{\prime \prime}$ of $Y^{\prime}$ without Euclidean factors, $\rho(\Gamma)$ stabilizes no points in $Y^{\prime \prime}(\infty)$, the sphere at infinity of $Y^{\prime \prime}$, i.e., is not contained in a parabolic subgroup of $I\left(Y^{\prime \prime}\right)$. When $Y$ is a symmetric space of noncompact type so that $I(Y)$ is a linear algebraic group $G$, this reduces to the usual definition of reductivity, that is, that the unipotent radical of the Zariski closure of $\rho(\Gamma)$ is trivial. In geometric terms, reductivity excludes the possibility that there exists an unbounded sequence $\left(y_{n}\right) \subset Y^{\prime \prime}$ with $d\left(y_{n}, \rho(\gamma) y_{n}\right) \leq c(\gamma)$ for all $\gamma \in \Gamma$, with some constant $c(\gamma)$ that may depend on $\gamma$, but not on $n$. This in turn prevents a minimizing sequence for the energy to disappear at infinity. A simple example where reductivity does not hold and the limit of a minimizing sequence does not exist is provided by a hyperbolic Riemann surface $N$ with a cusp and a homotopy class of maps $S^{1} \rightarrow N$ represented by a short noncontractible loop around the cusp. Such a homotopy class then is nontrivial, but does not contain a closed geodesic, that is, no harmonic map. The corresponding homomorphism $\rho: \pi_{1}\left(S^{1}\right)=\mathbb{Z} \rightarrow I(Y)$ (where $Y$ now is the Poincaré upper half plane $H$, and $I(Y)=P S L(2, \mathbb{R})$ ) has a parabolic image and is therefore not reductive. This shows that the reductivity condition is necessary for the existence of a harmonic map. We have $([36,49])$

THEOREM 1. Let $\rho: \Gamma \rightarrow I(Y)$ be a reductive homomorphism into the isometry group of a simply connected, complete Riemannian manifold of nonpositive sectional curvature. Assume that there exists some $\rho$-equivariant map $f: X \rightarrow Y$ with $E(f)<\infty$ (see (2.6)). Then there also exists a smooth $\rho$-equivariant harmonic map $h: X \rightarrow Y$ of finite energy. 
Moreover, when $Y$ has strictly negative curvature, this map is unique, unless it is of rank $\leq 1$ in which case the image is a point or a geodesic in $Y$.

When the fundamental domain for $\Gamma$ in $X$ is not bounded, or in a different formulation, when $X / \Gamma$ is not compact, then the existence of a finite energy map $f$ as needed for the theorem can become a difficult issue. For some conditions where such a finite energy map on noncompact domains can be constructed, see $[\mathbf{3 4}, \mathbf{3 5}]$. Alternatively, one may try to produce harmonic maps of infinite energy. For maps between Riemann surfaces, this was achieved by Lohkamp [54] and Wolf [83]. For the higher dimensional case, see $[\mathbf{3 8}]$. Applications of infinite energy harmonic maps to Teichmüller theory, however, will be presented elsewhere.

2.1.2. Harmonic and conformal maps from Riemann surfaces. The energy integral, and therefore harmonic maps, have some special property when the dimension of the domain is 2 , because of conformal invariance, as we are now going to explain. For this discussion, we assume that the quotients considered in the previous section are manifolds, that is, without singularities. In order to emphasize that two-dimensional domains are special, we denote the domain by $S$ and let it be an oriented two-dimensional Riemannian manifold with metric $\gamma_{\alpha \beta}$. By the uniformization theorem of Gauss, $S$ then obtains the structure of a Riemann surface, that is, we can locally find holomorphic coordinates $z=x+i y$ on $S$ such that the metric tensor becomes

$$
\lambda^{2}(z) d z \otimes d \bar{z}
$$

where $\lambda(z)$ is a real valued positive function. Conversely, given a compact Riemann surface $\Sigma$, by the Poincaré uniformization theorem, we can find a conformal metric as in (2.9) with constant curvature; we shall be mainly interested in the case of genus $\geq 2$, the hyperbolic case, where this curvature is negative, -1 . This metric is unique (up to diffeomorphism, see below). As such a surface $S$ is of the form $H / \Gamma$, where $H$ is the upper half plane $H:=\{x+i y \in \mathbb{C}: y>0\}$ and $\Gamma$ is a discrete subgroup of the isometry group of $H, P S L(2, \mathbb{R})$, this metric on $S$ descends from the hyperbolic metric $\frac{1}{y^{2}} d z \otimes d \bar{z}$ on $H$.

Let $N$ be a Riemannian manifold with metric tensor $\left(g_{i j}\right)$. The energy of a map $f: \Sigma \rightarrow N$ then becomes

$$
\begin{aligned}
E(f) & =\frac{1}{2} \int_{\Sigma} \frac{4}{\lambda^{2}(z)} g_{i j} \frac{\partial f^{i}}{\partial z} \frac{\partial f^{j}}{\partial \bar{z}} \frac{i}{2} \lambda^{2}(z) d z \wedge d \bar{z} \\
& =\int_{\Sigma} g_{i j} \frac{\partial f^{i}}{\partial z} \frac{\partial f^{j}}{\partial \bar{z}} i d z \wedge d \bar{z} .
\end{aligned}
$$

The last equation implies that the energy of a map from a Riemann surface $\Sigma$ into a Riemannian manifold is conformally invariant in the sense that it does not depend on the choice of a metric on $\Sigma$, but only on the Riemann surface 
structure. Also, if $k: \Sigma_{1} \rightarrow \Sigma_{2}$ is a bijective holomorphic or antiholomorphic map between Riemann surfaces then for any $f: \Sigma_{2} \rightarrow N$ (of class $C^{1}$ )

$$
E(f \circ k)=E(f),
$$

and if $f$ is harmonic, then so is $f \circ k$.

Likewise, the harmonic map equation is independent of the choice of conformal metric on the domain. This means that the map $h: S_{1} \rightarrow N$ of class $C^{2}$ is harmonic iff

$$
\frac{\partial^{2} h^{i}}{\partial z \partial \bar{z}}+\Gamma_{j k}^{i}(h(z)) \frac{\partial h^{j}}{\partial z} \frac{\partial h^{k}}{\partial \bar{z}}=0 \quad \text { for } i=1, \ldots, \operatorname{dim} N .
$$

When the target is also a Riemann surface $S_{2}$ with metric

$$
\rho^{2}(h) d h \otimes d \bar{h},
$$

then the harmonic map equation becomes

$$
h_{z \bar{z}}+\frac{2 \rho_{h}}{\rho} h_{z} h_{\bar{z}}=0 .
$$

Thus, holomorphic or antiholomorphic maps between Riemann surfaces are harmonic as they obviously satisfy (2.14). In the sequel, we shall write \pm holomorphic to mean holomorphic or antiholomorphic. The converse does not necessarily hold. Harmonic maps exist in any homotopy class of maps between compact Riemann surfaces, but \pm holomorphic maps $h: S_{1} \rightarrow S_{2}$ have to satisfy the Riemann-Hurwitz formula

$$
2-2 p_{1}=|m|\left(2-2 p_{2}\right)-v_{h},
$$

where $p_{1}, p_{2}$ are the genera of $S_{1}, S_{2}, m$ is the degree of $h$, and $v_{h} \geq 0$ is its total ramification index. In particular, as a necessary topological condition for a harmonic map to be \pm holomorphic, we have the inequality

$$
\chi\left(S_{1}\right) \leq|m| \chi\left(S_{2}\right),
$$

$\chi$ denoting the Euler characteristic. In fact, even if (2.16) holds, in general there will not exist a holomorphic map between $S_{1}$ and $S_{2}$, as this requires that the conformal structure of $S_{1}$ be a conformal cover of the one of $S_{2}$. Thus, in general, harmonic maps between Riemann surfaces $S_{1}, S_{2}$ cannot be \pm holomorphic. Also, as we see from (2.12), whether a map is harmonic depends on the metric of the target $S_{2}$ whereas the property of being \pm holomorphic only depends on the conformal structure of $S_{2}$, but not on its metric. Nevertheless, harmonic maps into Riemann surfaces enjoy special properties, as demonstrated by the following result of Schoen-Yau [68] and Sampson [66].

TheOREM 2. Let $h: S_{1} \rightarrow S_{2}$ be a harmonic map with $p_{1}=p_{2},|\operatorname{deg} h|=$ 1. If the curvature of $S_{2}$ satisfies $K_{2} \leq 0$, then $h$ is a diffeomorphism. 
(In fact, harmonic diffeomorphism exist even without the curvature restriction, see $[\mathbf{1 2}, \mathbf{3 2}, \mathbf{4 1}]$.) A key ingredient in the proof are the following identities. We put, for a harmonic map $h: S_{1} \rightarrow S_{2}$ between Riemann surfaces with curvatures $K_{1}, K_{2}$,

$$
H:=|\partial h|^{2}:=\frac{\rho^{2}}{\lambda^{2}} h_{z} \bar{h}_{\bar{z}} \text { and } L:=|\bar{\partial} h|^{2}:=\frac{\rho^{2}}{\lambda^{2}} h_{\bar{z}} \bar{h}_{z} .
$$

Then, at points where $H$ resp. $L$ is nonzero,

$$
\begin{aligned}
-\Delta \log H & =2 K_{1}-2 K_{2}(H-L) \\
-\Delta \log L & =2 K_{1}+2 K_{2}(H-L) .
\end{aligned}
$$

Actually, the Riemann-Hurwitz formula (2.15) can also be deduced from such identities, noting that for instance when $h$ is holomorphic, $L \equiv 0$.

The relationship between harmonic and \pm holomorphic maps (or conformal maps, when the target is of higher dimension) is clarified by the following:

Lemma 1. Let $\Sigma$ be a Riemann surface, $N$ a Riemannian manifold. If $h: \Sigma \rightarrow N$ is harmonic, then

$$
\varphi(z) d z^{2}=\left\langle\frac{\partial h}{\partial z}, \frac{\partial h}{\partial z}\right\rangle_{N} d z^{2}
$$

is a holomorphic quadratic differential. Furthermore, $\varphi(z) d z^{2} \equiv 0$ iff $h$ is conformal.

This is proved by an easy computation.

Returning to the case where the target is also a Riemann surface $S$, that is, where we have a map $h: \Sigma \rightarrow S$, we obtain, with the above notation $\lambda^{2} d z d \bar{z}$ and $\rho^{2} d h d \bar{h}$ for the metrics on $\Sigma$ and $S$,

$$
\rho^{2} d h d \bar{h}=\varphi(z) d z^{2}+\lambda^{2}(H+L) d z d \bar{z}+\bar{\varphi}(z) d \bar{z}^{2} .
$$

(Of course, when we recall the definition (2.17) of $H$ and $L$, the domain metric $\lambda^{2} d z d \bar{z}$ drops out. Also, in our local coordinates, $\varphi(z) d z^{2}=\rho^{2} h_{z} \bar{h}_{z} d z^{2}$.) Thus, the quadratic differential $\varphi(z) d z^{2}$ is the $(2,0)$-part of the pullback of the image metric.

In intrinsic terms, a (holomorphic) quadratic differential is a (holomorphic) section of $T_{\mathbb{C}}^{*} \Sigma \otimes T_{\mathbb{C}}^{*} \Sigma$. Since every holomorphic quadratic differential on the 2 -sphere $S^{2}$ vanishes identically, Lemma 1 implies

Corollary 1. For any Riemannian manifold $N$, every harmonic map

$$
h: S^{2} \rightarrow N
$$

is conformal. 


\subsection{Moduli spaces of Riemann surfaces.}

2.2.1. The algebro-geometric structure. In the realm of algebraic geometry, one naturally employs a terminology that refers to the complex dimension; thus, a smooth holomorphic curve is a Riemann surface, and a smooth algebraic surface is a complex manifold of complex dimension 2 that can be embedded into some complex projective space. Of course, there are some fundamental theorems behind this identification of algebraic objects - algebraic curves - and complex analytic - Riemann surfaces - and differential geometric ones - differentiable surfaces with certain metrics. We shall now briefly recall those results to prepare the stage for our discussion of moduli spaces from the various different perspectives.

A compact Riemann surface can be locally described as the common zero set of two homogeneous polynomials in three variables, that is, each compact Riemann surface carries the conformal structure of such a polynomial zero locus. Therefore, it becomes an algebraic variety in the projective space $\mathbb{C P}^{3}$. The Riemann surface is thus represented as the zero set of algebraic equations (and therefore called an algebraic curve). Such equations of a given degree can be characterized by their coefficients. However, different coefficient sets need not necessarily lead to different Riemann surfaces. In fact, automorphisms of $\mathbb{C P}^{3}$ lead to equivalent algebraic curves. Therefore, one needs to divide them out. For this, however, one encounters the difficulty that the automorphism group of $\mathbb{C P}^{3}$ is not compact. Mumford $[60,61]$ was able to overcome this difficulty. He developed geometric invariant theory to obtain the moduli space $M_{p}$ of algebraic curves of genus $p$. The moduli space of algebraic curves of a given genus is not compact. Algebraic equations can degenerate, and in geometric terms, limits of smooth algebraic curves can be singular. In order to obtain a compact moduli space, one therefore has to include such limits. In order to stay with smooth curves, one takes the singularities out. The resulting curves then are no longer compact. A problem emerges when such limits possess a noncompact automorphism group, like the once or twice punctured sphere. When one includes such objects, the resulting moduli space will lose its Hausdorff property. Therefore, Mumford constructed a moduli space that only consists of stable curves, that is, curves with a compact automorphism group. From this construction, the compactified Mumford-Deligne moduli space $\bar{M}_{p}$ is obtained as the moduli space of stable curves of genus $p$, see [14]. As a moduli space of algebraic varieties, it is an algebraic variety itself. Here, stable curves can have singularities. Such singularities correspond to double points of the algebraic curves. Therefore, on the algebraic curve, they come in pairs. If one takes out these singular points, one obtains noncompact curves with punctures. Let us look at the case of a single singularity, that is, where two points are taken out. Such a curve can either be connected of genus $p-1$, or it can consist of two components whose genera satisfy $p_{1}+p_{2}=p$. Here, all such components of genus 0 have to be ignored, as they would have noncompact automorphism groups. The process can be 
iterated, that is, limits of curves with one singularity may have two or more singularities. Consequently, we need to take out $2 k$ points from such curves, for some positive integer $k$, with the restriction again that the resulting curve be stable, that is, excluding spheres with one or two punctures. As boundary points in $\bar{M}_{p}$, such a curve then is determined by its conformal structure plus the positions of the punctures. The moduli space $M_{p}$ itself has complex dimension $3 p-3$, and the largest compactification strata have dimension $3 p-4$. For instance, for a compactification stratum corresponding to curves of genus $p-1$ with two punctures, we have $3(p-1)-3$ dimensions for the conformal structure and two dimensions for the positions of the two punctures, which yields $3 p-4$ altogether. The smallest compactification stratum corresponds to a sphere with 3 punctures, and it therefore is of dimension 0 , because all thrice punctured spheres are conformally equivalent.

We can also interpret the different compactification strata as follows. A surface with a singularity is conformally a compact surface minus the points corresponding to the singularity. Here, "singularity" could mean a double point, but we could also view it as two points at infinity. When we add these two points, without identifying them, that is, without creating a double point, we obtain a compact surface with two distinguished points. In general, surfaces of genus $p$ with $k$ distinguished points also are parametrized by a moduli space $M_{p, k}$ whose construction is completely analogous to the one of $M_{p}$. Thus, the Mumford-Deligne compactification of $M_{p}$ contains several compactification strata of the form $M_{p-1,2}, M_{p-2,4}, \ldots$ or $M_{p_{1}, 1} \cup M_{p_{2}, 1}$ with $p_{1}+p_{2}=p$, and so on. That is, $\bar{M}_{p}$ is naturally a stratified space, as already explained.

There exists a different way to compactify the moduli space $M_{p}$. This arises by associating to each algebraic curve its Jacobian variety. Since this correspondance is injective by Torelli's theorem, one can view $M_{p}$ as a subvariety of the moduli space $A_{p}$ of Abelian varieties of dimension $p$. The latter possesses a compactification $\bar{A}_{p}$, constructed by Satake and BailyBorel. This then induces a compactification $\overline{\bar{M}}_{p}$ of $M_{p}$ that was studied by Baily[5]. $\bar{M}_{p}$ is different from $\bar{M}_{p}$ and, in fact, highly singular, but it can simply be obtained from $\bar{M}_{p}$ by forgetting the positions of the punctures or cusps of the limiting Riemann surfaces in $\bar{M}_{p}$. Whereas the largest compactification strata of $\bar{M}_{p}$ have codimension 1 , those of $\overline{\bar{M}}_{p}$ have codimension 3 in $M_{p}$, with the exception of one stratum, corresponding to once punctured tori, that has codimension 2 . In any case, we should note that $M_{p}$ is not a manifold as it possesses some quotient singularities, arising from algebraic curves with nontrivial automorphism groups. A similar observation applies to the various compactification strata of $\bar{M}_{p}$. From the point of view of alge-

braic geometry, these singularities are rather mild. In contrast, the space $\overline{\bar{M}}_{p}$ is highly singular at the compactification locus. 
The moduli space $M_{p}$ can also be obtained from the general theory of variations of complex structures, as a universal space for variations of complex structures on a 1-dimensional complex manifold of genus $p$. This means that if $N$ is a complex space fibering over some base $B$ with the generic (=regular) fiber being a Riemann surface of genus $p$, we then obtain a holomorphic map $h: B_{0} \rightarrow M_{p}$ where $B_{0} \subset B$ are the points with regular fibers. In this manner, $M_{p}$, as a moduli space of complex structures, acquires a complex structure itself that is determined by the requirement that all these $h$ be holomorphic. Ideally, we would also like to have a so-called fine moduli space $M_{p, \text { fine }}$. This means that from the holomorphic map $h$, we obtain a holomorphic map $h_{\text {fine }}: N_{0} \rightarrow M_{p, \text { fine }}, N_{0}$ being the space of regular fibers in $N$, that maps the fiber over $w \in B_{0}$ to the fiber over $h(w)$ in $M_{p, f i n e}$. Again, the Riemann surfaces with non-trivial automorphisms cause difficulties, and such a fine moduli space does not exist. A slight modification, however, leads to such a fine moduli space; namely, we only need to equip our Riemann surfaces additionally with some choice of a root of the canonical bundle in order to prevent non-trivial automorphisms. This is called a level structure. This gives a finite ramified cover $M_{p}^{\prime}$ of $M_{p}$. That cover is free of singularities and then yields a fine moduli space $\mathcal{M}^{\prime}$ whose fiber over $w \in M_{p}^{\prime}$ is the Riemann surface (with level structure) determined by $w$.

Also, the cotangent space of $M_{p}$ at the point corresponding to the Riemann surface $\Sigma$ is naturally identified with the holomorphic quadratic differentials on $\Sigma$, that is, with the holomorphic sections of $T_{\mathbb{C}}^{*} \Sigma \otimes T_{\mathbb{C}}^{*} \Sigma$. We also recall that by the Riemann-Roch theorem, the complex dimension of that vector space is $3 p-3$ (see e.g., [45]).

2.2.2. Teichmüller theory. Again, we are mainly interested in Riemann surfaces of genus $p \geq 2$. As already mentioned, such a Riemann surface $\Sigma$ is a quotient $H / \Gamma$ of the Poincaré upper half plane by a discrete group $\Gamma \in P S L(2, \mathbb{R})$ of isometries with respect to the hyperbolic metric $\frac{1}{y^{2}} d z \otimes d \bar{z}$, with $z=x+i y$. Since $\Gamma$ is isomorphic to the fundamental group $\pi_{1}(S)$, the Riemann surface is described by a faithful representation $\rho$ of $\pi_{1}(S)$ in $P S L(2, \mathbb{R})$. This leads to the approach of Ahlfors and Bers to Teichmüller theory. Representations that only differ by a conjugation with an element of $P S L(2, \mathbb{R})$ yield the same conformal structure. Thus, we consider the space of faithful representations up to conjugacy. A representation can be defined by the images of the generators, that is, by $2 p$ elements of $G$, and this induces a natural topology on the moduli space. Also, from an easy count, we see that the (real) dimension of the moduli space of representations of $\pi_{1}(\Sigma)$ in $\operatorname{PSL}(2, \mathbb{R})$ modulo conjugations is $6 p-6$. This, of course, agrees with the dimension of the moduli space $M_{p}$ above. In fact, the moduli space presently discussed of discrete, faithful representations of $\pi_{1}(\Sigma)$ in $\operatorname{PSL}(2, \mathbb{R})$ modulo conjugations yields the Teichmüller space $T_{p}$, a simply connected singularityfree infinite cover of $M_{p}$. $M_{p}$ then is obtained as the quotient of $T_{p}$ by the mapping class group $\Gamma_{p}$. $\Gamma_{p}$ is the group of homotopy classes of positively 
oriented diffeomorphisms of the underlying surface $S$, or expresses somewhat differently,

$$
\Gamma_{p}=\operatorname{Diff}^{+}(S) / \operatorname{Diff}_{0}(S),
$$

where Diff ${ }^{+}$stands for orientation preserving diffeomorphisms and Diff for those that are homotopic to the identity of $S$.

As a moduli of representations of a discrete group, $T_{p}$ also acquires natural structures, like a differentiable and a complex one. It is diffeomorphic, but not biholomorphic to $\mathbb{C}^{3 p-3}$. The complex structure of Teichmüller space was first investigated systematically studied by Ahlfors [2]. A fundamental contribution is due to Bers who holomorphically embedded $T_{p}$ as a bounded domain in some complex Banach space, see $[\mathbf{6}]$ and the detailed presentation in [62]. Recently, higher Bers maps have been constructed by Buss[8].

2.2.3. The Riemannian approach. Since a two-dimensional Riemannian manifold defines a conformal structure, that is, a Riemann surface, we can naturally look at all Riemannian metrics on a given compact surface $F$ of genus $p$ and then identify those that induce the same conformal structure. We shall describe here the approach of Tromba and Fischer, see [77]. Thus, we consider the space $R_{p}$ of all smooth Riemannian metrics on $F . R_{p}$ has been studied in $[\mathbf{1 1}, \mathbf{1 5}, \mathbf{1 7}, \mathbf{1 8}, \mathbf{2 0}, \mathbf{2 1}]$, to cite some of the most significant contributions. This space carries itself a Riemannian metric. To describe this metric on $R_{p}$, let $g=\left(g_{i j}\right)_{i, j=1,2}$ (in local real coordinates $\left(z^{1}, z^{2}\right)$ ) be some Riemannian metric on $F$, that is, $g$ is an element of $R_{p}$. $\left(g_{i j}\right)$ is a positive definite symmetric $2 \times 2$ tensor. Tangent vectors to $R_{p}$ at $g$ then are given by symmetric $2 \times 2$ tensors $\left(h_{i j}\right),\left(\ell_{i j}\right)$. The metric of $R_{p}$ on the tangent space at $g$ is then given by

$$
\left(\left(h_{i j}\right),\left(\ell_{i j}\right)\right)_{g}:=\int_{F} \operatorname{trace}(h, \ell) d v o l(g)=\int_{F} g^{i j} g^{k m} h_{i k} \ell_{j m} \sqrt{\operatorname{det} g} d z^{1} d z^{2} .
$$

This $L^{2}$-metric on the infinite dimensional space $R_{p}$ is only a weak Riemannian metric, that is, the tangent spaces of $R_{p}$ are not complete w.r.t. this metric. Therefore, the general theory of Riemann-Hilbert manifolds does not apply. Nevertheless, Clarke $[\mathbf{1 1}]$ showed $R_{p}$ becomes a metric space with the distance function induced by this weak metric (2.23). Moreover, Clarke [10] was able to identify the metric completion of this metric space. Here, one needs to allow for semimetrics, that is, give up the requirement of strict positive definiteness, and then identify two such semimetrics that only differ at points where neither of them is positive definite.

Of course, we are interested in metrics only up to isometry. That is, when two metrics $g_{1}, g_{2}$ are isometric to each other, then they are considered as the same metric. This, however, is not yet reflected in the definition of $R_{p}$. Thus, we need to go to a quotient of $R_{p}$ that is obtained by identifying isometric metrics. If $g$ is a Riemannian metric on $F$ and $h: F \rightarrow F$ is a diffeomorphism, $h^{\star} g$ is isometric to $g$ via $h$. Conversely, when two metrics $g, h$ are isometric to 
each other, then the isometry between them is given by some diffeomorphism of $F$. Thus, we need to divide out the action of the (orientation preserving) diffeomorphism group $D_{p}$ of $F . D_{p}$ acts isometrically on $R_{p}$ equipped with its Riemannian metric.

In addition to this issue, also two metrics that are not isometric might still induce the same conformal structure, and since we are interested in the latter, we should also identify all metrics that induce the same conformal structure. When we multiply a given metric $g$ by some positive function $\lambda$, the metric $\lambda g$ leads to the same conformal structure as $g$. Conversely, when two metrics are conformally equivalent, they are related by such a positive function.

One then gets rid of the ambiguity of the conformal factor by finding a suitable slice in $R_{p}$ transversal to the conformal changes. By Poincaré's theorem, any Riemannian metric on our surface $S$ of genus $p>1$ is conformally equivalent to a unique hyperbolic metric, that is, $S$ becomes a quotient $H / \Gamma$ as above. This metric has constant curvature -1 . The moduli space $M_{p}$ is then obtained as the space $R_{p,-1}$ of metrics of curvature -1 divided by the action of $D_{p}$. In this way, the geometric structures on $R_{p}$ induce corresponding geometric structures on $M_{p}$ as described in [77]. A tangent vector to $R_{p}$, that is a symmetric $2 \times 2$ tensor $\left(h_{i j}\right)$, is orthogonal to the conformal multiplications when it is trace-free, and it is orthogonal to the action of $D_{p}$ when it is divergence-free. Such a trace- and divergence-free symmetric tensor then can be identified with a holomorphic quadratic differential on the Riemann surface.

The Riemannian metric on $R_{p}$ then induces a Riemannian metric on the moduli space $M_{p}$, or more precisely, on its singularity free covers like $T_{p}$, as developed in [19]. This metric then induces a product between holomorphic quadratic differentials on the Riemann surface $S$ in question. We use a complex notation. Let $\varphi, \psi$ be holomorphic quadratic differentials on $S$, and denote the hyperbolic metric on $S$ by $\lambda^{2} d z d \bar{z}$. Their product w.r.t. the metric then is given by

$$
(\varphi, \psi)_{g}=2 \operatorname{Re} \int \varphi \bar{\psi} \frac{1}{\lambda^{2}(z)} d z d \bar{z} .
$$

This metric is called the Weil-Petersson metric $g_{W P}$. This metric was introduced by Weil inspired constructions of Petersson in number theory. Ahlfors $\left[\begin{array}{l}1,2 \\ 2\end{array}\right.$ derived basic results about this metric. In particular, the WeilPetersson is a Kähler metric w.r.t. the natural complex structure of the moduli space. This metric is not complete, since $\partial \bar{M}_{p}:=\bar{M}_{p} \backslash M_{p}$ has finite distance from the interior, as discovered by Wolpert [85]. Masur [58] then found precise expansions of the Weil-Petersson metric near the boundary of $M_{p}$. Masur's estimates yield detailed information about the metric completion of $M_{p}$ with the Weil-Petersson metric. The remarkable result is that this metric completion agrees with the Mumford-Deligne compactification of $M_{p}$ discussed above. 
While the Weil-Petersson metric is not complete, it possesses strictly convex exhaustion functions. Such functions were found by Wolpert[87] and Tromba $[\mathbf{7 8 , 7 9}]$. Tromba's function is of particular interest in the present context because it is given by the energy functional for harmonic maps between Riemann surfaces as a function of the conformal structure of the domain.

Yamada [90] observed that the existence of strictly convex exhaustion function implies that geodesics starting in the interior of $M_{p}$ can hit the boundary at most at their endpoints. In other words, a geodesic between two points in a compactification stratum has to stay inside that stratum. This fact has been extended to harmonic maps by Wentworth[80].

For a recent review of the geometry of the completion of the WeilPetersson metric, see $[\mathbf{8 8}]$.

Tromba [75] proved that $g_{W P}$ has negative sectional curvature, and its holomorphic sectional curvature even has a negative upper bound $k<0$. Different proofs of these results were found in $[\mathbf{3 1}, \mathbf{4 2}, \mathbf{7 0}, \mathbf{8 6}]$. For a recent survey of the curvature properties of the Weil-Petersson metric, see [89]. The curvature of the Weil-Petersson metric is not bounded negatively from above, as was first shown by Huang [27] who found that the curvature goes to 0 in certain directions when approaching the boundary of moduli space. This issue has been further investigated in $[\mathbf{2 9}, \mathbf{7 4}]$. Also, in [56] it was shown that the Weil-Petersson metric is not Gromov hyperbolic. For further results, see [7].

The isometry group of the Weil-Petersson metric is given by the extended mapping class group, as shown in [57], where extended means that one takes all diffeomorphisms, and not only the orientation preserving ones in (2.22). After going to finite covers, as explained above, but without changing our notation for simplicity, we have a holomorphic fibering

$$
\psi: \mathcal{M}_{p}^{\prime} \rightarrow M_{p}^{\prime}
$$

where the fiber over $q \in M_{p}^{\prime}$ is the holomorphic curve defined by $q$. $\mathcal{M}_{p}^{\prime}$ also carries a Weil-Petersson metric with the same negativity properties as the one of $M_{p}^{\prime}$, see e.g., [42].

Similarly, at the level of Teichmüller spaces, we have the universal Teichmüller curve $\mathcal{T}_{p}$, the fiber bundle over $T_{p}$ where the fiber over a point in $T_{p}$ is the marked Riemann surface represented by that point. Of course, we then have a covering map $\mathcal{T}_{p} \rightarrow \mathcal{M}_{p}^{\prime}$. In particular, $\mathcal{T}_{p}$ also carries its Weil-Petersson metric of negative curvature.

The Weil-Petersson metric employs the hyperbolic metric $\lambda^{2} d z d \bar{z}$ on the Riemann surface $S$. One can then replace this hyperbolic metric by another natural metric on $S$, like the Bergmann or the Arakelov metric. This was investigated in $[\mathbf{2 4}, \mathbf{2 5}, \mathbf{2 8}, \mathbf{3 0}]$.

Besides these metrics and the Weil-Petersson metric, Teichmüller space carries many other natural metrics, including the Teichmüller, Kobayashi and Caratheodory metrics which are only Finsler metrics, the Bergman and 
Kähler-Einstein metrics. More recently, Liu-Sun-Yau[50, 51, 52] introduced new metrics, the Ricci metric which is given by the negative Ricci tensor of the Weil-Petersson metric and the perturbed Ricci metric where a multiple of the Weil-Petersson metric is added. They study the curvature and boundary behavior of these metrics and achieve a systematic comparison of all the aforementioned metrics.

\section{Harmonic maps to Riemann surfaces}

3.1. Harmonic maps between Riemann surfaces. We consider harmonic maps $h: \Sigma \rightarrow S$ between compact Riemann surfaces of hyperbolic type, that is, of genus $\geq 2$. We assume that they both have the same genus $p$. $S$ needs to carry some Riemannian metric, and unless stated otherwise, we take the hyperbolic metric of constant curvature -1 .

As explained in Section 2.1.2, the harmonic map $h$ induces a holomorphic quadratic differential on $\Sigma$, that is, a holomorphic section of $T_{\mathbb{C}}^{*} \Sigma \otimes T_{\mathbb{C}}^{*} \Sigma$. Thus, from the harmonic map $h$, we obtain a cotangent vector of the moduli space $M_{p}$ at the point corresponding to $\Sigma$. It is then natural to investigate how this cotangent vector varies when we vary the conformal structure of either $\Sigma$ or $S$. Let us consider the effect of variations of the latter. This will depend on the following summary of Theorems 1 and 2 and Lemma 1.

Lemma 2. Let $\Sigma$ be a compact Riemann surface with local conformal coordinate $z=x+i y, S$ another such surface of the same genus $p \geq 2$, equipped with its unique hyperbolic metric, locally written as $\rho^{2}(h) d h d \bar{h}$. We also fix some (orientation preserving) diffeomorphism $k: \Sigma \rightarrow S$. Then there exists a unique harmonic map

$$
h=h(\Sigma, S): \Sigma \rightarrow S
$$

homotopic to $k$. This map $h$ is a diffeomorphism,

$$
\varphi(z) d z^{2}=\rho^{2} h_{z} \bar{h}_{z} d z^{2}
$$

is a holomorphic quadratic differential, and $h$ is conformal iff $\varphi=0$.

We keep $\Sigma$ fixed. $S$ then determines the harmonic map $h$ which in turns yields a holomorphic quadratic differential on $\Sigma$. Denoting the space of those holomorphic quadratic differentials by $Q(\Sigma)$, we then obtain a map

$$
q(\Sigma): T_{p} \rightarrow Q(\Sigma)
$$

The map $q$ is defined on Teichmüller space $T_{p}$ instead of on the moduli space $M_{p}$ because the harmonic map depends on the choice of a diffeomorphism $k: \Sigma \rightarrow S$. Wolf [81] (see also [43]) then showed

TheOREM 3. For any $\Sigma, q(\Sigma)$ is a bijection between $T_{p}$ and $Q(\Sigma)$. 
The proof makes crucial use of the identities (2.18), (2.19).

Wolf $[\mathbf{8 2}]$ then also analyzed the asymptotic behavior of this bijection. His striking result is that this yields the Thurston compactification of Teichmüller space that had originally been constructed in a very different manner. Geometrically, one sees from [84] how harmonic maps between surfaces converge to harmonic maps from a surface to an $\mathbb{R}$-tree when the target surface degenerates in the manner described above. Minsky [59] then provided estimates about energy and extremal length which describe the asymptotics as the source decays.

Theorem 3 says that with its natural differentiable structure, $T_{p}$ is diffeomorphic to the vector space $\mathbb{C}^{3 p-3}$ which is Teichmüller's theorem. It is, however, not biholomorphic to that space with respect to its natural complex structure (see [43] for a detailed analysis of this issue). Also, $q(\Sigma)$ is not an isometry w.r.t. the Weil-Petersson metric on $T_{p}$. Nevertheless, there does exist a relationship between harmonic maps and the Weil-Petersson metric that we are now going to describe. For that purpose, we consider the energy

$$
E(h(\Sigma, S))=\int_{\Sigma} \rho^{2}(h(z))\left(h_{z} \bar{h}_{\bar{z}}+h_{\bar{z}} \bar{h}_{z}\right) d z d \bar{z}=\int_{\Sigma}(H+L) \lambda^{2}(z) d z d \bar{z}
$$

as a function of $S$ and present some formulas obtained in [43]. When $S=\Sigma$ as an element of $T_{p}$, that is, when the conformal structure of $S$ is such that $k$ is homotopic to a conformal diffeomorphism - which then is given by the harmonic map $h(\Sigma, S)$-, then $E$ as a function of $S$ achieves its minimum. This can be seen, for instance, from the fact that when $h$ is conformal, then $L=0$, and the integral of the Jacobian,

$$
J(h)=\int_{\Sigma}(H-L) \lambda^{2}(z) d z d \bar{z},
$$

is a topological invariant that does not depend on the conformal structures of $\Sigma$ and $S$, but only on their genus. At the point $S=\Sigma \in T_{p}$, the infinitesimal variations of the target surface correspond to the cotangent vectors of $T_{p}$ at $\Sigma$, that is, to the holomorphic quadratic differentials on $\Sigma$. Since $E$ achieves a minimum here, we have

$$
E_{\varphi}=0 \text { for all } \varphi \in Q(\Sigma)
$$

where $E_{\varphi}=\frac{d}{d t} E(h(\Sigma, S+t \varphi))$ is the derivative of $E$ for a variation of the target in the direction of $\varphi$, and where we implicitly use the identification of $T_{p}$ with $Q(\Sigma)$ given by Theorem 3 . In fact,

LEMma 3. $S=\Sigma$ is the only critical point of $E$ as a function of $S \in T_{p}$. Moreover, $E(h(\Sigma,)$.$) is a proper function on T_{p}$. Its second derivatives at $S=\Sigma$ for $\varphi, \psi \in Q(\Sigma)$ are given by

$$
E_{\varphi \bar{\psi}}=2 \int_{\Sigma} \varphi \bar{\psi} \frac{1}{\lambda^{2}} d z d \bar{z}
$$


Consequently, $E(h(\Sigma,)$.$) is a proper exhaustion function with a single$ critical point at which its Hessian is positive definite.

The same result holds when we consider $E(h(\Sigma, S))$ as a function of the domain $\Sigma$, as proved by Tromba [76], who, by appealing to elementary Morse theory, had thus found a harmonic map proof that Teichmüller space is topologically a cell. We should also mention the detailed and useful computations of Yamada [90] here. For systematic computations of the variations of the energy as a function of both the domain and the target, see $[43]$.

The important interpretation of (3.7) is that the second variation of the energy at $S=\Sigma$ yields the Weil-Petersson product of the holomorphic quadratic differentials $\varphi, \psi$. This can be used for systematic expansions [42].

Let us also briefly mention that harmonic maps can be used as a tool to understand grafting, see $[\mathbf{1 6}, \mathbf{6 7}, \mathbf{7 3}]$.

\subsection{Harmonic maps from Kähler manifolds to Riemann sur-} faces. Our starting point is the discovery of $[\mathbf{3 3}]$.

THEOREM 4. Let $M$ be a compact Kähler manifold, $S$ a hyperbolic Riemann surface, $h: M \rightarrow S$ harmonic. If $d h\left(w_{0}\right) \neq 0$ at some $w_{0} \in M$, then in some neighborhood $U$ of $w_{0}$, the local level sets

$$
\{h=z\} \cap U, \quad(z \in S)
$$

consist of complex analytic hypersurfaces, and the corresponding foliation is holomorphic.

This holomorphic foliation then induces a holomorphic equivalence, that is, by identifying the points in the level set, one concludes (see [71])

TheOREM 5. Let $M$ be a compact Kähler manifold, $S$ a compact hyperbolic Riemann surface, $g: M \rightarrow S$ continuous and homologically nontrivial, that is,

$$
g_{*}: H_{2}(M, \mathbb{R}) \rightarrow H_{2}(S, \mathbb{R})
$$

is nontrivial. Then there exist a compact hyperbolic Riemann surface $S^{\prime}$, a holomorphic map $u: M \rightarrow S^{\prime}$, and a harmonic map $\phi: S^{\prime} \rightarrow S$ for which the harmonic map $h:=\phi \circ u$ is homotopic to $g$.

Thus, if $D$ is the hyperbolic unit disk, the lift

$$
\tilde{u}: \tilde{M} \rightarrow D
$$

is a nonconstant bounded holomorphic function on $\tilde{M}$.

In other words, the harmonic map $h: M \rightarrow S$ factors through some holomorphic map $u: M \rightarrow S^{\prime}$ to another Riemann surface $S^{\prime}$ that is obtained as the quotient of $M$ by the holomorphic foliation of Theorem 4. In general, $S^{\prime}$ 
is not only conformally, but also topologically different from $S$. We consider the example where $S$ is a compact hyperbolic surface of genus $2, M$ is a compact hyperbolic surface $\Sigma$ of genus 3 , and $g$ is a map of degree 1 that collapses one of the handles of $\Sigma$ to a point. Since there does not exist a holomorphic map of degree 1 from a surface of genus 3 to one of genus 2 , $S^{\prime}$ must be topologically different from $S$. In fact, in this example, $S^{\prime}=\Sigma$, and the holomorphic map $u$ is simply the identity. Thus, when $M$ is itself a Riemann surface, the statement of Theorem 5 is trivial. It becomes quite important, however, for higher dimensional Kähler manifolds. It implies, in particular, that the existence of a nontrivial holomorphic map to a (compact, singularity free) holomorphic curve of genus $\geq 2$ is a topological property of a Kähler manifold. We shall see an application in Section 4.

\section{Harmonic and holomorphic maps to moduli spaces}

In this section, we shall describe the second method of applying harmonic maps to moduli and Teichmüller spaces. The first method consisted in investigating how harmonic maps between Riemann surfaces depend on the underlying conformal structure. For instance, by looking at the energies of such harmonic maps, we could construct useful functions on moduli or Teichmüller spaces. The second method consists rather in looking at harmonic maps to moduli or Teichmüller spaces. As described in Section 2.2, these spaces carry geometric structures, including a natural Kähler metric of negative curvature, in their own right, and so, we can also directly consider them as potential target spaces for harmonic mappings.

By doing so, we can then also appeal to general results about when harmonic maps are \pm -biholomorphic, as started in [69]. This method is very useful because harmonic maps exist under rather general circumstances, as described in Section 2.1.1, and therefore one possesses a powerful tool for construction holomorphic maps. Conversely, holomorphic maps are special cases of harmonic maps, and therefore also succumb to general results about the latter.

We shall first describe how harmonic map methods apply to families of Riemann surfaces. Here, a family of Riemann surfaces is described as a subvariety of some $\bar{M}_{p}$. We have the theorem of Parshin [64] (the case $D=$ $\emptyset)$ and Arakelov [3] (general $D$ ), the solution of the Shafarevitch problem over function fields:

TheOrem 6. Let $C$ be a compact smooth holomorphic curve, $D \subset S$ finite, and let $g \geq 2$. Then there exist at most finitely many algebraic surfaces $B$ fibered over $C$ with smooth fibres of genus $g$ over $C \backslash D$ that are not isotrivial, that is, not finitely covered by a product.

Translated into the language of mappings, this means that there exists a holomorphic map $f: B \rightarrow C$ for which $f^{-1}(z)$ is a smooth holomorphic curve of genus $g$ for every $z \in C \backslash D$. The setting can then be generalized. 
THEOREM 7. Let $Z$ be a compact manifold, $D \subset Z$ a divisor with at most simple normal crossings. Then there exist at most finitely many nonisotrivial fiber spaces over $Z$ for which the fibers over the points in $Z \backslash D$ are smooth curves of genus $g$.

The next result is due to Manin [55] and Grauert [23], the solution of the Mordell problem over function fields.

TheOREM 8. Let $f: B \rightarrow C$ be a nontrivial fibering as in Theorem 6 . Then there exist at most finitely many holomorphic sections $s: C \rightarrow B$.

Again, this result readily generalizes as in Theorem 7 .

The harmonic map approach to these results that we are going to describe was developed in [37]. As described in Section 2.2.1, $\bar{M}_{g}$ satisfies some universal property. This property is that fiberings $f: B \rightarrow C$ by curves of genus $\mathrm{g}$ as in Theorem 6 give rise to holomorphic maps $h: C \rightarrow \bar{M}_{g}$ by associating to $z \in C \backslash D$ the holomorphic structure of the curve $f^{-1}(z)$ and by then extending this map to all of $C$. In order to avoid the quotient singularities of $M_{p}$ and to distinguish isotrivial families, we lift to finite covers (as described in Section 2.2.1, $h^{\prime}: C^{\prime} \rightarrow M_{g}^{\prime}$. $h^{\prime}$ is constant iff the fibering is isotrivial. Equipping $C^{\prime}$ with a metric of constant curvature $\kappa$ and $M_{g}^{\prime}$ with the Weil-Petersson metric whose holomorphic sectional curvature has an upper bound $k<0$ (see Section 2.2.3), the Schwarz Lemma of Yau [91] and Royden [65] yields

$$
\left\|d h^{\prime}(z)\right\|^{2} \leq \frac{\kappa}{k} \text { for all } z \in C^{\prime},
$$

unless $h^{\prime}$ is constant. If the genus of $C$ is 0,1 , then $\kappa \geq 0$, and so, in this case,we conclude that any fibering $f: B \rightarrow C$ by curves of genus $\geq 2$ is isotrivial, because then the right hand side of (4.1) is nonpositive. For the case where the genus of $C$ is $\geq 2$, by (4.1), all holomorphic maps $h^{\prime}$ : $C^{\prime} \rightarrow M_{g}^{\prime}$ are equicontinuous. With an additional technical argument needed to handle the geometry of the boundary of compactified moduli space, one then concludes that at most finitely many homotopy classes of maps from $C^{\prime}$ to $\bar{M}_{g}^{\prime}$ can contain holomorphic maps. This yields the so-called boundedness part of the proof of Theorem 6 (for a related argument, see $[\mathbf{2 2}, \mathbf{6 3}]$ ). For the second step of the proof, called finiteness, consists in showing that any nontrivial homotopy class of maps from $C^{\prime}$ to $\bar{M}_{g}^{\prime}$ can contain at most one harmonic and therefore in particular at most one holomorphic map. This again follows from the negativity of the curvature of $\bar{M}_{g}^{\prime}$. For the proof of Theorem 8, we utilize the holomorphic fibering

$$
\psi: \mathcal{M}_{g}^{\prime} \rightarrow M_{g}^{\prime}
$$

and recall from Section 2.2.3 that $\mathcal{M}_{g}^{\prime}$ also carries a Weil-Petersson metric with the same negativity properties as the one of $M_{g}$. A holomorphic section 
$s$ as in Theorem 8 then induces a holomorphic map $k: C^{\prime} \rightarrow \mathcal{M}_{g}^{\prime}$. The scheme of proof then can be based on the same strategy as the one of Theorem 6 . Also, it is clear that this scheme also extends to higher dimensional bases as in Theorem 7 .

Moreover, this scheme can be converted into quantitative height and Arakelov type inequalities, as opposed to mere qualitative finiteness theorems, see $[\mathbf{4 0 , 5 3}]$.

Also, one can show the existence of fibrations and other holomorphic families by turning the scheme around. That is, one first shows the existence of an appropriate harmonic map into $M_{g}$ or $T_{g}$ and then appeals to Siu's theorem [69] or some variant of it to show that this harmonic map is in fact holomorphic. There exist two variants of this strategy (see [37] for details):

(1) Let $M$ be a compact Kähler manifold, $A \subset M$ a divisor with at most simple normal crossings as singularities, $f: M \rightarrow \bar{M}_{g}$ a continuous map that is locally liftable near the quotient singularities of $\bar{M}_{g}$ and that is not homotopic to a map into $\bar{M}_{g} \backslash M_{g}$ or to a map of real rank $\leq 2$ everywhere. We then produce an equivariant harmonic map $h: \widetilde{M \backslash A} \rightarrow T_{g}$ by some variant of Theorem 1, equipping $T_{g}$ with its negatively curved Weil-Petersson metric. In fact, there is the technical issue here that this metric is not complete, as discussed above. However, for the existence scheme to go through it suffices that the target possess some strictly convex exhaustion function. As discussed in Section 2.2.3, such functions exist on $T_{g}$. And when one has such a strictly convex exhaustion function, one can control the escape of harmonic maps to infinity by the maximum principle, see [35]. The reason is that the composition of a harmonic map with a convex function is subharmonic which is easily shown by direct computation, see e.g. [46]. (A more precise result about the control of images of harmonic maps in terms of the different strata of compactified moduli space $\bar{M}_{g}$ has been derived by Wentworth [80].) Thus, one can show the existence of some equivariant harmonic map $h: \widetilde{M \backslash A} \rightarrow T_{g}$, see [35] (we do not discuss here the technical issue of the finiteness of the energy). And since the Weil-Petersson has strongly negative curvature in the sense of [69], this harmonic map then has to be \pm holomorphic. We can then pull back the universal Teichmüller curve $\mathcal{T}_{g}$ over $T_{g}$ to $M \backslash A$ via that holomorphic map to obtain a fibration by holomorphic curves over $M \backslash A$. Under appropriate assumptions, this then extends to all of $M$.

In the same spirit, one can construct holomorphic maps $h: \widetilde{M \backslash A} \rightarrow$ $\mathcal{T}_{g}$, in order to obtain fibrations of $M \backslash A$ by holomorphic curves of genus $g$. 
(2) Let $B$ be a compact Kähler surface, $C$ a compact curve, $f: B \rightarrow$ $C$ a continuous map that is nontrivial on the second homology, $A$ a divisor with at most simple normal crossings on $B, S \subset C$ finite for which $C \backslash S$ carries a complete hyperbolic metric, such that $f$ maps $B \backslash A$ to $C \backslash S$. By the strategy of Theorems 4,5 , one then constructs a holomorphic map $h$ from $B \backslash A$ to some curve $C^{\prime} \backslash S^{\prime}$ that in turn maps harmonically to $C \backslash S$. By the topological assumptions, the fibers of $h$ are of complex dimension 1, that is, holomorphic curves. Thus, we can construct holomorphic fibrations of Kähler surfaces from topological assumptions. Similar methods apply in higher dimension, when we have an appropriate map $f$ from a Kähler manifold of dimension $m$ to another of dimension $m-1$.

Most known nontrivial compact holomorphic families of stable holomorphic curves contain some singular elements, that is, they do not yield maps from the base to moduli space $M_{g}$, but only to its compactification $\bar{M}_{g}$. One important example of such a family without singular fibers is given by the Kodaira surfaces that were introduced by Kodaira [48], rediscovered by Atiyah [4] and further investigated by Hirzebruch [26]. The construction starts with a compact Riemann surface $R_{0}$ of genus $g_{0} \geq 2$, and then a twosheeted unramified covering of $R_{0}$, which is a Riemann surface $R$ of genus $g=2 g_{0}-1$. Let $\tau$ be the fixed point free automorphism of $R$ corresponding to that covering. We have a canonical homomorphism from the fundamental group $\pi_{1}(R)$ to $H_{1}\left(R, \mathbb{Z}_{m}\right)=\left(Z_{m}\right)^{2 g}$. We then obtain an $m^{2 g}$-sheeted unramified covering surface $v: S \rightarrow R$ of genus $h=m^{2 g}(g-1)+1$ of $R$ by mapping $\pi_{1}(S)$ to the kernel of that homomorphism. We consider the graphs $\Gamma_{v}$ and $\Gamma_{\tau \circ v}$ and the divisor $D=\Gamma_{v}-\Gamma_{\tau \circ v}$. The integral homology class of $D$ then is divisible by $m$. This allows us to construct an algebraic surface $M$ which is a covering of $R \times S$ ramified along the preimage of $D$. This is the Kodaira surface. In [33] it was shown with harmonic map techniques that every deformation of a Kodaira surface is again such a surface, and that the deformation space is given by the moduli space of unramified double coverings of a Riemann surface of genus $g_{0}$. (The statement in $[\mathbf{3 3}]$ was not completely correct; it has been corrected in [37].) For recent progress on Kodaira surfaces, see $[\mathbf{9}]$ and the references therein.

We now return to the situation of Theorem 5 . We consider a smooth analytic local family $M_{t}, t \in T$ of Kähler manifolds over some not necessarily compact local base $T$, with $M_{0}=M$. That is, we consider deformations of $M$. Since $M$ admits a topologically nontrivial map $g: M \rightarrow S$ to some higher genus Riemann surface, so then then do its deformations $M_{t}$. Consequently, we get a family of holomorphic maps

$$
u_{t}: M_{t} \rightarrow S_{t}^{\prime}
$$

to Riemann surfaces, of some genus $p \geq 2$. 
TheOREM 9. The local family of holomorphic maps $u_{t}, t \in T$ is holomorphic. That is, we obtain an induced holomorphic map

$$
U: T \rightarrow M_{p}
$$

into the moduli space of Riemann surfaces of genus $p$.

Proof. First of all, the family is well defined, since the harmonic maps $h_{t}: M_{t} \rightarrow S$ and holomorphic maps $u_{t}: M_{t} \rightarrow S_{t}^{\prime}$ are unique in their homotopy classes, because they are topologically nontrivial and the target is hyperbolic, see Theorem 1. Also, the family is continuous, because, for instance, the holomorphic foliation of Theorem 4 and hence also its quotient $S_{t}^{\prime}$ depends continuously on $t$, as can be seen from [33]. Alternatively, one can appeal to general functional analytic results $[\mathbf{6 6}]$ about the smooth dependence of harmonic maps on the geometries of domain and target, again in the presence of negative target curvature.

That the family is holomorphic then reduces to an infinitesimal argument. We simply need to show that the corresponding infinitesimal variation of the family is holomorphic, that is, $X_{t}:=\frac{\partial u_{t}}{\partial t}=0$, and w.l.o.g., it suffices to consider this at $M_{0}=M$. We can use the argument of Kalka [47] here (for a more abstract argument, see $[\mathbf{3 9}]$ ). The local family $S_{t}$ maps into the universal Teichmüller curve $\mathcal{T}_{p}$. This family possesses a metric of strongly negative curvature in the sense of $[\mathbf{6 9}]$. This follows, for instance, from the fact that $\mathcal{T}_{p}$ can be identified with the Teichmüller space $T_{p, 1}$ of once punctured marked Riemann surfaces of genus $p$, and the latter carries its negatively curved Weil-Petersson metric, as described in Section 2.2.3. Thus, we obtain a map $V:\left\{M_{t}\right\}_{t \in T} \rightarrow \mathcal{T}_{p}$ whose restriction $v_{t}$ to any fiber is holomorphic. As observed by Kalka [47], the infinitesimal version $\frac{\partial v_{t}}{\partial t}$ then is a holomorphic section of a negative holomorphic bundle, hence vanishes. This means that $X_{t}=0$, and our family is holomorphic.

In fact, by appealing to Spallek's theorem [72], we only need to assume here that $T$ is a holomorphic space germ, not necessarily smooth.

Therefore, we can also look at the global situation where $T$ is the moduli space for the Kähler manifold $M$, assuming that such a moduli space exists. We assume that there is no nontrivial monodromy, that is, there does not exist a closed loop in $T$ such that when following the target surfaces $S_{t}^{\prime}$ around this loop leads to a Riemann surface that is different from the one that the loop started with. This is a topological condition on the maps $M_{t} \rightarrow$ $S$, that is, their homotopy class does not have a nontrivial monodromy along a closed loop in $T$. Under these conditions, when there exists a topologically nontrivial continuous map $g: M \rightarrow S$ to some compact hyperbolic surface of genus $p$, we obtain a meromorphic map

$$
U: T \rightarrow M_{p} .
$$




\section{References}

[1] L. Ahlfors. Curvature properties of Teichmüller's space. Journ. d'Anal. Math., 9:161-176, 1961.

[2] L. Ahlfors. Some remarks on Teichmüller's space of Riemann surfaces. Ann. Math., 74:171-191, 1961.

[3] S. Arakelov. Families of algebraic curves with fixed degeneracies. Math. USSR Izv., 5:1277-1302, 1971.

[4] M. Atiyah. The signature of fibre bundles. In D.C. Spencer and S. Iyanaga, editors, Global analysis. Princeton Univ. Press, 1969.

[5] W. Baily. On the moduli of Jacobian varieties. Ann. Math., 71:303-314, 1960.

[6] L. Bers. A non-standard integral equation with applications to quasiconformal mappings. Acta Math., 116:113-134, 1966.

[7] J. Brock and B. Farb. Curvature and rank of Teichmüller space. Amer. J. Math., 128:1-22, 2006.

[8] G. Buss. Higher Bers maps. arXiv:0812.0314, 2008.

[9] F. Catanese and S. Rollenske. Double Kodaira fibrations. arxiv:math/0611428v1, 2006.

[10] B. Clarke. The completion of the manifold of Riemannian metrics. arXiv:0904.0177, 2009.

[11] B. Clarke. The metric geometry of the manifold of Riemannian metrics over a closed manifold. arXiv:0904.0174, 2009.

[12] J.M. Coron and F. Hélein. Harmonic diffeomorphisms, minimizing harmonic maps and rotational symmetry. Compos. Math., 69:175-228, 1989.

[13] G. Daskalopoulos and R. Wentworth. Harmonic maps and Teichmüller theory. In: Handbook of Teichmüller theory. Vol. I (A. Papadopoulos, ed.), pages 33-109. EMS, 2007.

[14] P. Deligne and D. Mumford. The irreducibility of the space of curves of given genus. Publ. Math. IHES, 36:75-110, 1969.

[15] B. DeWitt. Quantum theory of gravity. I. The canonical theory. Phys. Rev., 160:1113$1148,1967$.

[16] D. Dumas. Grafting, pruning, and the antipodal map on measured laminations. J. Differential Geom., 74:93-118, 2006.

[17] D. Ebin. The manifold of Riemannian metrics. In S.S. Chern and S. Smale, editors, Global analysis, volume 15 of Proc. Symp. Pure Math., pages 11-40. AMS, 1970.

[18] A. Fischer and A. Tromba. On a purely Riemannian proof of the structure and dimension of the unramified moduli space of a compact Riemann surface. Math. Ann., 267:311-345, 1984.

[19] A. Fischer and A. Tromba. On the Weil-Petersson metric on Teichmüller space. Trans. $A M S, 284: 319-335,1984$.

[20] D. Freed and D. Groisser. The basic geometry of the manifold of Riemannian metrics and of its quotient by the diffeomorphism group. Mich. Math. Journ., 36:323-344, 1989.

[21] O. Gil-Medrano and P. Michor. The Riemannian manifold of all Riemannian metrics. Quart. J. Math. Oxford Ser., 42:183-202, 1991.

[22] H. Grauert and H. Reckziegel. Hermitesche Metriken und normale Familien holomorpher Abbildungen. Math. Z., 89:108-125, 1965.

[23] H. Grauert. Mordells Vermutung über rationale Punkte auf algebraischen Kurven und Funktionenkörper. Publ. Math. IHES, 25:363-381, 1965.

[24] L. Habermann and J. Jost. Riemannian metrics on Teichmüller space. man. math., 89:281-306, 1996.

[25] L. Habermann and J. Jost. Metrics on Riemann surfaces and the geometry of moduli spaces. In J. P. Bourguignon, P. di Bartolomeis, and M. Giaquinta, editors, Geometric 
theory of singular phenomena in partial differential equations, Cortona 1995, pages 53-70. Cambridge Univ. Press, Cambridge, 1998.

[26] F. Hirzebruch. The signature of ramified coverings. In D.C. Spencer and S. Iyanaga, editors, Global analysis. Princeton Univ. Press, 1969.

[27] Z. Huang. Asymptotic flatness of the Weil-Petersson metric on Teichmüller space. Geom. Dedicata, 110:81-102, 2005.

[28] Z. Huang. Calculus of Variations and the L2-Bergman Metric on Teichmüller Space. arXiv:math.DG/0506569, 2006.

[29] Z. Huang. The Weil-Petersson geometry on the thick part of the moduli space of Riemann surfaces. Proc. AMS, 135:3305-3316, 2007.

[30] J. Jorgenson and J. Kramer. Non-completeness of the Arakelov-induced metric on moduli space of curves. Man. math., 119:453-463, 2006.

[31] J. Jost and X.W. Peng. Group actions, gauge transformations and the calculus of variations. Math. Ann., 293:595-621, 1992.

[32] J. Jost and R. Schoen. On the existence of harmonic diffeomorphisms between surfaces. Inv. math., 66:353-359, 1982.

[33] J. Jost and S.T. Yau. Harmonic mappings and Kähler manifolds. Math. Ann., 262:145-166, 1983.

[34] J. Jost and S.T. Yau. The strong rigidity of locally symmetric complex manifolds of rank one and finite volume. Math. Ann., 275:291-304, 1986.

[35] J. Jost and S.T. Yau. On the rigidity of certain discrete groups and algebraic varieties, Math. Ann. 278 (1987), 481-496. Math. Ann., 278:481-496, 1987.

[36] J. Jost and S.T. Yau. Harmonic maps and group representations. In H.B. Lawson and K. Tenenblat, editors, Differential Geometry, pages 241-259. Longman Scientific, 1991.

[37] J. Jost and S.T. Yau. Harmonic maps and algebraic varieties over function fields. Amer. J. Math., 115:1197-1227, 1993.

[38] J. Jost and K. Zuo. Harmonic maps of infinite energy and rigidity results for Archimedean and non-Archimedean representations of fundamental groups of quasiprojective varieties. J. Diff. Geom., 47:469-503, 1997.

[39] J. Jost and K. Zuo. Representations of fundamental groups of algebraic manifolds and their restrictions to fibers of a fibration. Math. Res. Letters, 8:569-575, 2001.

[40] J. Jost and K. Zuo. Arakelov type inequalities for Hodge bundles over algebraic varieties, Part I: Hodge bundles over algebraic curves. J. Alg. Geom., 11:535-546, 2002 .

[41] J. Jost. Univalency of harmonic mappings between surfaces. J. reine angew. Math., 324:141-153, 1981.

[42] J. Jost. Harmonic maps and curvature computations in Teichmüller theory. Ann. Acad. Sci. Fen., Series A. I. Math., 16:13-46, 1991.

[43] J. Jost. Two-dimensional geometric variational problems. Wiley-Interscience, 1991.

[44] J. Jost. Harmonic mappings. In L.Z. Ji et al., editor, Handbook of Geometric Analysis, pages 147-194. International Press, 2008.

[45] J. Jost. Compact Riemann surfaces. Springer, ${ }^{3} 2006$.

[46] J. Jost. Riemannian geometry and geometric analysis. Springer, ${ }^{5} 2008$.

[47] M. Kalka. Deformation of submanifolds of strongly negatively curved manifolds. Math. Ann., 251:243-248, 1980.

[48] K. Kodaira. A certain type of irregular algebraic surfaces. J. Anal. Math., 19:207-215, 1967.

[49] F. Labourie. Existence d'applications harmoniques tordues à valeurs dans les variétés à courbure négative. Proc. Am. Math. Soc., 111:877-882, 1991.

[50] K.-F. Liu, X.F. Sun, and S.T. Yau. Canonical metrics on the moduli space of Riemann surfaces, I. Journ. Diff. Geom., 68:571-637, 2004. 
[51] K.-F. Liu, X.F. Sun, and S.T. Yau. Canonical metrics on the moduli space of Riemann surfaces, II. Journ. Diff. Geom., 69:163-216, 2005.

[52] K.-F. Liu, X.F. Sun, and S.T. Yau. Geometric aspects of the moduli space of Riemann surfaces. Science in China Ser. A: Math., 48(Supp.1):97-122, 2005.

[53] K.-F. Liu. Geometric height inequalities. Math. Res. Letters, 3:637-702, 1996.

[54] J. Lohkamp. An existence theorem for harmonic maps. Man. math., 67:21-23, 1990.

[55] J. Manin. Rational points of algebraic curves over function fields. Izv. Akad. Nauk. SSSR, Ser. Mat., 27:1395-1440, 1963.

[56] H. Masur and M. Wolf. Teichmüller space is not Gromov hyperbolic. Ann. Acad. Sci. Fenn., 20:259-267, 1995.

[57] H. Masur and M. Wolf. The Weil-Petersson isometry group. Geom. Dedicata, 93:177190, 2002.

[58] H. Masur. The extension of the Weil-Petersson metric to the boundary of Teichmüller space. Duke Math. J., 43:623-635, 1977.

[59] Y.N. Minsky. Harmonic maps, length, and energy in Teichmüller space. J. Differential Geom., 35:151-217, 1992.

[60] D. Mumford. Geometric invariant theory. Springer, 1965.

[61] D. Mumford. Stability of projective varieties. L'Enseignement Math., 23:39-110, 1977.

[62] S. Nag. The complex analytic theory of Teichmüller spaces. Wiley, 1988.

[63] J. Noguchi. Moduli spaces of holomorphic mappings into hyperbolically imbedded complex spaces and locally symmetric spaces. Inv. Math., 93:15-34, 1988.

[64] A.N. Parshin. Algebraic curves over function fields, i. Math. USSR Izv., 2:1145-1170, 1968.

[65] H. Royden. The Ahlfors-Schwarz lemma in several complex variables. Comment. Math. Helv., 55:558-574, 1980.

[66] J. Sampson. Some properties and applications of harmonic mappings. Ann. Sci. Ecole Normale Superieure, 11:211-228, 1978.

[67] K.P. Scannell and M. Wolf. The grafting map of Teichmüller space. J. Amer. Math. Soc., 15:893-927, 2002.

[68] R. Schoen and S.T. Yau. On univalent harmonic maps between surfaces. Inv. Math., 44:265-278, 1978.

[69] Y.T. Siu. The complex analyticity of harmonic maps and the strong rigidity of compact Kähler manifolds. Ann. Math., 112:73-111, 1980.

[70] Y.T. Siu. Curvature of the Weil-Petersson metric in the moduli space of compact Kähler-Einstein manifolds of negative first Chern class. In K. Diederich, editor, Aspects of Mathematics, vol. 9, pages 261-298. Birkhäuser, 1987.

[71] Y.T. Siu. Strong rigidity for Kähler manifolds and the construction of bounded holomorphic functions. In R. Howe, editor, Discrete groups in geometry and analysis, pages 124-151. Birkhäuser, 1987.

[72] K. Spallek. Differenzierbare und holomorphe Funktionen auf analytischen Mengen. Math. Ann., 161:143-162, 1965.

[73] H. Tanigawa. Grafting, harmonic maps and projective structures on surfaces. J. Differential Geom., 47:399-419, 1997.

[74] L.P. Teo. The Weil-Petersson geometry of the moduli space of Riemann surfaces. Proc. AMS, 137:541-552, 2009.

[75] A. Tromba. On a natural connection on the space of almost complex structures and the curvature of Teichmüller space with respect to its Weil- Petersson metric. Man. Math., 56:475-479, 1986.

[76] A. Tromba. A new proof that Teichmüller space is a cell. Trans. Am. Math. Soc., 303:257-262, 1990.

[77] A. Tromba. Teichmüller theory in Riemannian geometry. Birkhäuser, 1992.

[78] A. Tromba. Dirichlet's energy on Teichmüller's moduli space and the Nielsen realization problem. Math. Zeitschrift, 222:451-464, 1996. 
[79] A. Tromba. Dirichlet's energy on Teichmüller's moduli space is strictly plurisubharmonic. In J. Jost, editor, Geometric analysis and the calculus of variations for Stefan Hildebrandt, pages 315-341. Intern.Press, 1996.

[80] R. Wentworth. Regularity of harmonic maps from Riemann surfaces to the WeilPetersson completion of Teichmüller space. 2004.

[81] M. Wolf. The Teichmüller theory of harmonic maps. J. Diff. Geom., 29:449-479, 1989.

[82] M. Wolf. High energy degeneration of harmonic maps between surfaces and rays in Teichmiller space. Topology, 30:517-540, 1991.

[83] M. Wolf. Infinite energy maps and degeneration of hyperbolic surfaces in moduli space. J. Diff. Geom., 33:487-539, 1991.

[84] M. Wolf. Harmonic maps from surfaces to R-trees. Math. Z., 218:577-593, 1995.

[85] S. Wolpert. Noncompleteness of the Weil-Petersson metric for Teichmüller space. Pacific J. Math., 61:513-576, 1975.

[86] S. Wolpert. Chern forms and the Riemann tensor for the moduli space of curves. Inv. Math., 85:119-145, 1986.

[87] S. Wolpert. Geodesic length functions and the Nielsen problem. J. Diff. Geom., 25:275-296, 1987.

[88] S. Wolpert. Geometry of the Weil-Petersson completion of Teichmüller space. arxiv, 0502528, 2005.

[89] S. Wolpert. Understanding Weil-Petersson curvature. to appear in Proc. Yau Birthday Conf; arxiv, 0809.3699, 2008.

[90] S. Yamada. Weil-Peterson convexity of the energy functional on classical and universal Teichmüller spaces. J. Differential Geom., 51:35-96, 1999.

[91] S.T. Yau. A general Schwarz lemma for Kähler manifolds. Amer. J. Math., 100:197203, 1978.

Max Planck Institute for Mathematics in the Sciences, 04103 Leipzig, Germany.

Math.Dept., Harvard Univ., Cambridge, MA 02138, USA. 